\title{
微波辅助 $\mathrm{SnCl}_{4} / \mathrm{H}_{2} \mathrm{SO}_{4}$ 二元体系催化纤维素醇解制备乙酰丙酸甲酯
}

\author{
黄耀兵杨涛刘安凤周新成潘 晖* \\ (南京林业大学化学与工程学院 南京 210037)
}

\begin{abstract}
摘要 主要研究了在微波条件下, 金属盐-酸混合二元体系催化纤维素醇解制备乙酰丙酸甲酯的反应. 在 $\mathrm{SnCl}_{4}$ 和 $\mathrm{H}_{2} \mathrm{SO}_{4}$ 的协同作用下, 纤维素被高效转化, 获得了 $61.5 \%$ 的乙酰丙酸甲酯的产率. 考察了催化剂比例、反应时间和反应 温度等参数对反应产率和选择性的影响, 研究了水的加入对于反应的促进作用. 同时, 将该体系应用到多种生物质原 料(如蔗糖、淀粉和菊粉等)转化为乙酰丙酸甲酯的反应中, 取得了 $48 \% \sim 60 \%$ 的产率. 催化剂经过 5 次的连续回收和重 复使用，依然保持了良好的催化活性.
\end{abstract}

关键词 纤维素; 乙酰丙酸甲酯; 微波辐射; 醇解反应

\section{Microwave-Assisted Alcoholysis of Cellulose to Methyl Levulinate Catalyzed by $\mathrm{SnCl}_{4} / \mathrm{H}_{2} \mathrm{SO}_{4}$}

\author{
Huang, Yaobing Yang, Tao Liu, Anfeng Zhou, Xincheng Pan, Hui* \\ ( College of Chemical and Engineering, Nanjing Forestry University, Nanjing 210037)
}

\begin{abstract}
Microwave irradiation technology was used to assist the conversion of cellulose to methyl levulinate (ML) in methanol solution in the presence of mixed catalysts. The reactions were carried out in a microwave reactor and cellulose was rapidly converted into ML with a highest yield of $61.5 \%$ by the use of $\mathrm{SnCl}_{4} / \mathrm{H}_{2} \mathrm{SO}_{4}$ as catalyst. The catalysts ratios, reaction time and reaction temperature were optimized and found to have great influences on both the ML product yield and the selectivity. Besides, an appropriate amount of water, which may participate the breakage of the glucosidic bond of cellulose, is highly desirable for the full conversion of cellulose. Several other biomass derived carbohydrates such as starch, sucrose and inulin were also tested in the optimized system and $48 \% \sim 60 \%$ ML yields were achieved. Finally, the mixed catalysts can be recycled and reused for at least five times without much decrease in catalytic activity.
\end{abstract}

Keywords cellulose; methyl levulinate; microwave irradiation; alcoholysis

随着化石资源的日益枯竭及其消耗过程中带来的 温室气体等环境问题, 越来越多的国家开始关注可再生 能源的开发利用. 在众多的可再生资源中, 生物质是唯 一的可再生的有机碳资源, 具有储量丰富、价格低廉的 特点. 实现生物质资源的高效、高品质利用具有广阔的 市场前景 ${ }^{[1]}$. 木质纤维素作为生物质资源的重要组成部 分, 通过水解方式可以将其高效地解聚制备可溶性糖类 分子(如葡萄糖、果糖等), 进一步转化则可以获得多种 平台分子, 如 5-羟甲基糠醛 ${ }^{[2,3]}$ 、乙酰丙酸 ${ }^{[4,5]}, \gamma$-戊内酯 等 ${ }^{[6]}$. 从这些平台化合物出发, 则可以转化得到众多的 化学品及燃料分子, 极大地拓展了生物质资源的应用领
域. 在这些分子中，乙酰丙酸酯是一类具有广泛应用前 景的有机平台化合物，可以用作塑化剂、溶剂、香料和 燃油添加剂等 ${ }^{[7]}$. 同时, 乙酰丙酸酯分子中的活性基团 (羰基和酯基)使其具有良好的反应活性，易通过加成、 取代、水解、酯交换等反应合成多种化学品和药品, 如 $\gamma$-戊内酯、吡咯烷酮等 ${ }^{[8 \sim 10]}$.

目前, 关于乙酰丙酸酯类的合成方法有许多报 道 ${ }^{[11,12]}$, 主要有三类: (1)乙酰丙酸的酯化. 使用酸催化 剂可以实现乙酰丙酸和醇在温和条件下的酯化得到目 标产物. 例如, Penedo 等 ${ }^{[13]}$ 使用了磺酸化介孔二氧化硅 $\left(\mathrm{SO}_{3} \mathrm{H}-\mathrm{SBA}-15\right)$ 催化剂，使用甲醇作为溶剂，在 $117{ }^{\circ} \mathrm{C}$

\footnotetext{
*E-mail: hyb@njfu.edu.cn, hpan@njfu.edu.cn

Received November 9, 2015; revised December 28, 2015; published online February 1, 2016.

Project supported by the National Natural Science Foundation of China (No. 21502095), the Natural Science Foundation of Jiangsu Province (No. BK20150872) and the Opening Fund from Jiangsu Key Laboratory of Biomass-based Green Fuels and Chemicals (No. 14003).

国家自然科学基金(No. 21502095)、江苏省自然科学基金(No. BK20150872)和江苏省生物质绿色燃料和化学品重点实验室开放基金(No. 14003)资助项 目.
} 
条件下反应 2 4 h 后，乙酰丙酸甲酯的产率可达 $95 \%$. 然而, 虽然以乙酰丙酸为初始原料的工艺简单且反应产 率高, 但反应需要较高纯度的乙酰丙酸, 价格等因素限 制了其在工业上的大规模应用. (2)糠醇的醇解. 使用酸 催化剂, 使糠醇和小分子醇发生醇解反应, 经开环后获 得乙酰丙酸酯. 反应原料糠醇则可通过大宗的糠醛加氢 获得, 因而这一反应更具应用潜力. 国内外众多课题组 报道了关于该反应的转化新体系 ${ }^{[11]}$. 本课题组 ${ }^{[14]}$ 曾报 道了使用金属盐催化糠醇醇解制备乙酰丙酸酯的新方 法. (3)碳水化合物发热醇解. 相比于上述两种方法, 以 葡萄糖、果糖或纤维素等碳水化合物为原料制备乙酰丙 酸酯则更具有优势. 近些年, 基于糖类化合物制备乙酰 丙酸酯的方法也取得了重要进展. 例如, 林鹿等 ${ }^{[15]}$ 以沉 淀-浸渍法合成了固体酸 $\mathrm{SO}_{4}^{2-} / \mathrm{SnO}_{2}$, 并将其用于催化 葡萄糖制备乙酰丙酸丁酯, 产率最高达 $33.1 \%$. Tsang 等 ${ }^{[16]}$ 制备了一系列的固体酸催化剂, 包括 $\mathrm{SO}_{4}^{2-} / \mathrm{TiO}_{2}$, $\mathrm{ZrO}_{2}, \mathrm{ZSM}-5$ 等. 在甲醇溶剂中, 以 $\mathrm{SO}_{4}^{2-} / \mathrm{TiO}_{2}$ 为催化 剂, 蔗糖、葡萄糖和果糖转化为乙酰丙酸甲酯的产率分 别为 $43 \%, 33 \%$ 和 $59 \%$.

为了进一步降低乙酰丙酸的生产成本, 以纤维素直 接醇解制备乙酰丙酸酯受到了广泛的关注. Tominaga 等 ${ }^{[17]}$ 报道了使用有机磺酸和高价 Lewis 酸盐的混合酸体 系实现了纤维素到乙酰丙酸甲酯的转化, 产率高达 $75 \%$. 李雪辉等 ${ }^{[18]}$ 使用磺酸功能化的离子液体 $\left(\left[\mathrm{C}_{4} \mathrm{H}_{8} \mathrm{SO}_{3} \mathrm{Hmim}\right] \mathrm{HSO}_{4}\right)$ 催化纤维素醇解获得 $31.1 \%$ 的乙 酰丙酸丁酯. 陈俊武等 ${ }^{\left[{ }^{[19}\right.}$ 报道了以超稳 $\mathrm{Y}$ 型分子篎 (USY)催化纤维素制备乙酰丙酸乙酯的转化. 最近, 刘 晓辉和王艳芹等 ${ }^{[20]}$ 提出了在磷酸铌固体酸催化下, 纤 维素可以 $56 \%$ 的产率转化得到乙酰丙酸甲酯. 上述方法 为纤维素直接醇解提供了很好的思路. 然而, 纤维素直
接醇解制备乙酰丙酸酯类的催化体系仍然相对较少，发 展新的体系仍然非常必要.

基于此, 本工作提出了使用质子酸 $\mathrm{H}_{2} \mathrm{SO}_{4}$ 和路易斯 酸金属盐 $\mathrm{SnCl}_{4}$ 的金属盐-酸二元混合体系, 在微波条件 下, 催化纤维素在醇溶剂中的醇解制备乙酰丙酸甲酯 (ML) (Scheme 1). 考察了催化剂种类、比例、反应温度、 时间和水用量等因素对反应产率和选择性的影响. 同 时, 对催化体系的循环利用特性也开展了相关的研究.

\section{1 结果与讨论}

\section{1 不同催化剂催化效果}

首先, 考察不同酸和金属盐的组合催化纤维素醇解 的反应效果(表 1). 选用甲醇作为溶剂, 当反应仅加入 $\mathrm{H}_{2} \mathrm{SO}_{4}$, 仍有 $43.8 \%$ 乙酰丙酸甲酯的生成(表 1, Entry 1). 这与前人报道的工作一致 ${ }^{[21]}$, 即在强酸存在的条件下, 纤维素仍可部分转化为乙酰丙酸酯. 而当只加入金属盐 的 $\mathrm{SnCl}_{4}$ 时, 在 $180{ }^{\circ} \mathrm{C}$ 反应条件下迅速碳化, 仅得到了 $3 \%$ 的 ML 产率, 并有少量的乳酸甲酯的生成(表 1, Entry 2). 这是由于 $\mathrm{SnCl}_{4}$ 具有较强的 Lewis 酸性, 在反应过程 中促进解聚后的单糖分子发生断键并异构化形成乳酸 甲酯(图 1). 当使用 $\mathrm{H}_{2} \mathrm{SO}_{4}$ 和 $\mathrm{SnCl}_{4}$ 共催化时, 产率提高 到 $61.5 \%$, 其分离产率也高达 $53.4 \%$. 当使用其他的金 属氯化物作为 Lewis 酸型催化剂时, 除了 $\mathrm{AlCl}_{3}$ 和 $\mathrm{CrCl}_{3}$ 取得了相对较好的效果外, 其余 $\mathrm{ZnCl}_{2}, \mathrm{FeCl}_{3}$ 和 $\mathrm{CuCl}_{2}$ 的 ML 均低于 $50 \%$. 由此可见，不同 Lewis 酸性金属盐 对反应有着较大的影响. 结合前人的工作, 我们推测 Lewis 酸性强弱关系到纤维素解聚后得到的甲基葡萄糖 苷的异构化速度, 从而影响了与后续的醇解反应的速率 匹配程度.

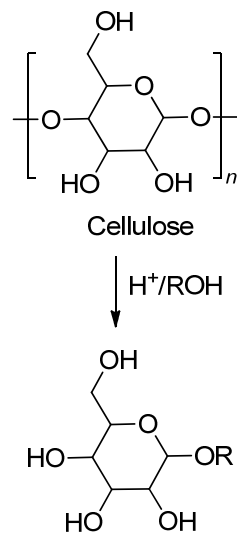

Alkyl glucoside

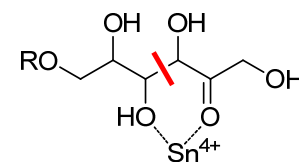<smiles>C#CC</smiles><smiles>COC(=O)C(C)O</smiles>

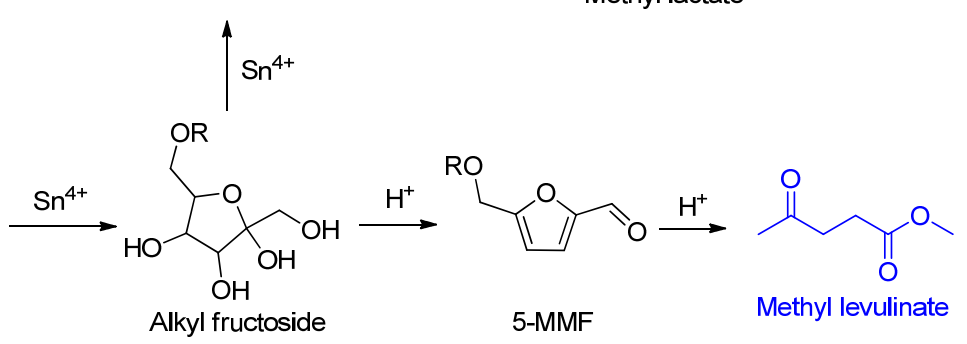

图式 1 纤维素醇解制备乙酰丙酸甲酯路径图

Scheme 1 Reaction pathway for the alcoholysis of cellulose into methyl levulinate 
表 1 不同质子酸和盐催化纤维素制备乙酰丙酸甲酯 ${ }^{a}$

Table 1 Conversion of cellulose to methyl levulinate with the addition of different acids and metal salts

\begin{tabular}{|c|c|c|c|c|}
\hline Entry & Acid & Metal salt & ML yield ${ }^{b} / \%$ & Methyl lactate yield ${ }^{b} / \%$ \\
\hline 1 & $\mathrm{H}_{2} \mathrm{SO}_{4}$ & - & 43.8 & 0 \\
\hline 2 & - & $\mathrm{SnCl}_{4}$ & 3.0 & 5.1 \\
\hline 3 & $\mathrm{H}_{2} \mathrm{SO}_{4}$ & $\mathrm{SnCl}_{4}$ & $61.5\left(53.4^{c}\right)$ & 4.8 \\
\hline 4 & $\mathrm{H}_{2} \mathrm{SO}_{4}$ & $\mathrm{FeCl}_{3}$ & 48.3 & 0.2 \\
\hline 5 & $\mathrm{H}_{2} \mathrm{SO}_{4}$ & $\mathrm{CuCl}_{2}$ & 46.2 & 0.5 \\
\hline 6 & $\mathrm{H}_{2} \mathrm{SO}_{4}$ & $\mathrm{AlCl}_{3}$ & 57.8 & 0.1 \\
\hline 7 & $\mathrm{H}_{2} \mathrm{SO}_{4}$ & $\mathrm{ZnCl}_{2}$ & 45.1 & 0 \\
\hline 8 & $\mathrm{H}_{2} \mathrm{SO}_{4}$ & $\mathrm{CrCl}_{3}$ & 56.5 & 1.1 \\
\hline 9 & $\mathrm{H}_{3} \mathrm{PO}_{4}$ & $\mathrm{SnCl}_{4}$ & 4.1 & 0 \\
\hline 10 & $\mathrm{HCl}$ & $\mathrm{SnCl}_{4}$ & 57.1 & 5.7 \\
\hline 11 & $\mathrm{H}_{3} \mathrm{O}_{40} \mathrm{PW}_{12} \bullet x \mathrm{H}_{2} \mathrm{O}$ & $\mathrm{SnCl}_{4}$ & 49.8 & 16.5 \\
\hline 12 & $\mathrm{H}_{4}\left[\mathrm{Si}\left(\mathrm{W}_{3} \mathrm{O}_{10}\right)_{4}\right] \cdot x \mathrm{H}_{2} \mathrm{O}$ & $\mathrm{SnCl}_{4}$ & 42.6 & 21.3 \\
\hline
\end{tabular}

${ }^{a}$ Condition: cellulose $500 \mathrm{mg}$, metal salt $0.2 \mathrm{mmol}$, acid $0.7 \mathrm{mmol}\left(\mathrm{H}^{+} 1.4 \mathrm{mmol}\right), \mathrm{H}_{2} \mathrm{O} 0.8 \mathrm{~mL}$, MeOH $14 \mathrm{~mL}, 800 \mathrm{~W}, 180{ }^{\circ} \mathrm{C}, 50 \mathrm{~min} ;{ }^{b} \mathrm{GC}$ yield. ${ }^{c}$ isolated yield.

此外，不同的酸对反应有较大的影响. 反应选取了 $\mathrm{H}_{3} \mathrm{PO}_{4}, \mathrm{HCl}$ 和杂多酸 $\left(\mathrm{H}_{3} \mathrm{O}_{40} \mathrm{PW}_{12} \bullet x \mathrm{H}_{2} \mathrm{O}, \mathrm{H}_{4}\left[\mathrm{Si}\left(\mathrm{W}_{3} \mathrm{O}_{10}\right)_{4}\right] \bullet\right.$ $x \mathrm{H}_{2} \mathrm{O}$ )进行反应测试(表 1). $\mathrm{H}_{3} \mathrm{PO}_{4}$ 对于纤维素的转化反 应的效率很低, 这是因为 $\mathrm{H}_{3} \mathrm{PO}_{4}$ 易与甲醇发生酯化反应 生成磷酸甲酯从而抑制了其酸的作用. 使用盐酸作为催 化剂也能得到较高的 ML 收率, 但盐酸的挥发性和腐蚀 性较强, 不利于催化剂的回收和实际应用 ${ }^{[22]}$. 使用杂多 酸 $\mathrm{H}_{3} \mathrm{O}_{40} \mathrm{PW}_{12} \cdot x \mathrm{H}_{2} \mathrm{O}$ 和 $\mathrm{H}_{4}\left[\mathrm{Si}\left(\mathrm{W}_{3} \mathrm{O}_{10}\right)_{4}\right] \cdot x \mathrm{H}_{2} \mathrm{O}$ 作为催化剂 可以分别得到 $49.8 \%$ 和 $42.6 \%$ 的 ML. 而乳酸甲酯的产率 却分别是 $16.5 \%$ 和 $21.3 \%$. 这可能是由于杂多酸分子结 构中的铇金属的 Lewis 酸性, 导致糖类中间体更多地发 生断键生成乳酸甲酯. 综上所述, $\mathrm{SnCl}_{4}$ 和 $\mathrm{H}_{2} \mathrm{SO}_{4}$ 组成的 混合体系是纤维素转化制备乙酰丙酸甲酯的最优催化 体系.

\section{$1.2 \mathrm{H}_{2} \mathrm{SO}_{4}$ 量对反应产率的影响}

$\mathrm{H}_{2} \mathrm{SO}_{4}$ 用量对反应的影响见图 2. 反应不加入硫酸 时, ML 的产率仅有 $3 \%$. 反应体系中出现了较多的黑色 沉淀, 可能是由于纤维素在高温下发生了碳化. 当加入

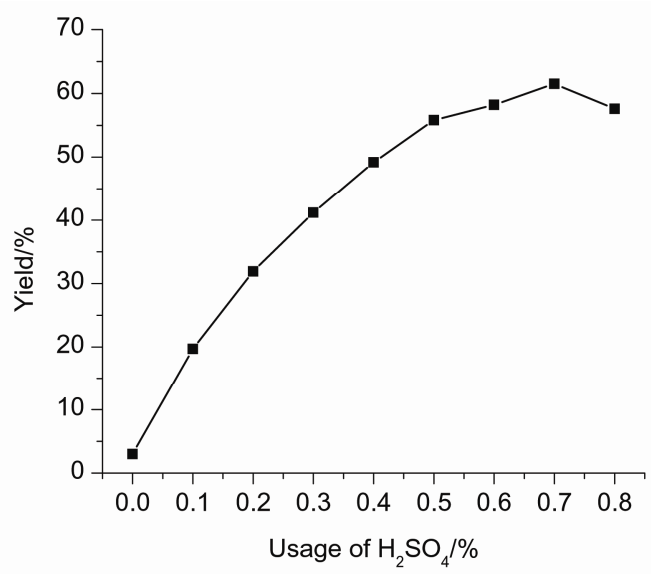

图 $2 \mathrm{H}_{2} \mathrm{SO}_{4}$ 的量对反应的影响

Figure 2 Influence of the amount of $\mathrm{H}_{2} \mathrm{SO}_{4}$
$3.3 \mathrm{~mol} \%$ 的硫酸时，乙酰丙酸甲酯的产率迅速提高到 19.7\%. 进一步增加硫酸的用量, ML 的产率进一步提高. 直到加入 $23.3 \mathrm{~mol} \%$ 硫酸时，反应获得了 $61.5 \%$ 的最高 产率. 过量的硫酸则会引起产率的轻微下降. 由此可以 看出, 合适的 $\mathrm{H}_{2} \mathrm{SO}_{4}$ 浓度可以有效促进纤维素的降解以 及后续的脱水醇解. 而过量的酸则很有可能会引起较多 的副反应，从而降低反应的产率 ${ }^{[23]}$.

\section{$1.3 \mathrm{SnCl}_{4}$ 量对反应产率的影响}

Lewis 酸性金属盐在纤维素的转化中也发挥着重要 作用. 如图 3 所示, 在不添加 $\mathrm{SnCl}_{4}$ 的情况下, $\mathrm{ML}$ 的产 率为 $43.8 \%$. 随着 $\mathrm{SnCl}_{4}$ 加入, $\mathrm{ML}$ 的产率得到了明显的 提高. 在 $\mathrm{SnCl}_{4}$ 用量为 $6.7 \mathrm{~mol} \%$ 时，产率达到最大值. 此 后，随着 $\mathrm{SnCl}_{4}$ 用量的继续增加，产物产率慢慢下降. 结 合前人对该反应的机理研究发现，在反应过程中，中间 体甲基果糖苷易在 $\mathrm{SnCl}_{4}$ 催化下发生 $\mathrm{C}-\mathrm{C}$ 键的断裂生 成乳酸甲酯，抑制了甲基果糖苷向 $\mathrm{ML}$ 的转化(图 1). 从 图中可以发现，随着 $\mathrm{SnCl}_{4}$ 用量增加，反应产生的

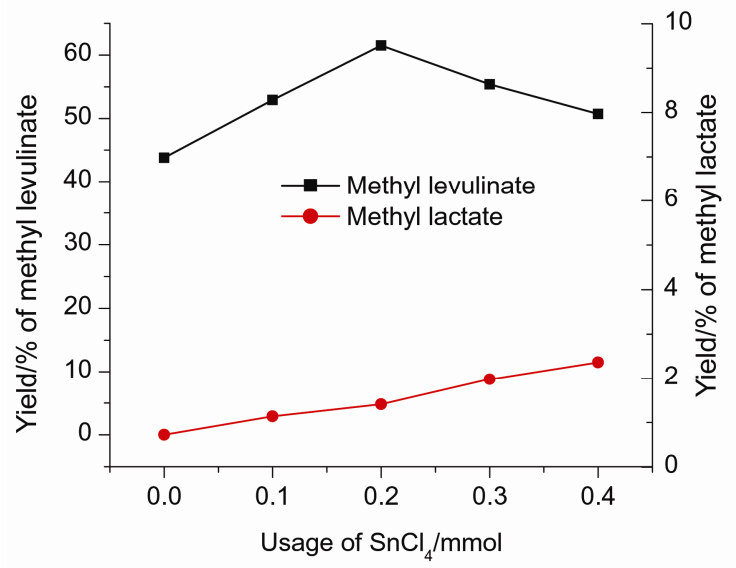

图 $3 \mathrm{SnCl}_{4}$ 的量对反应的影响

Figure 3 Influence of the amount of $\mathrm{SnCl}_{4}$ 
乳酸甲酯的量一直在上升. 因此, $\mathrm{SnCl}_{4}$ 用量需要进行优 化, 在获得最大目标产物产率的同时避免生成较多的乳 酸甲酯 ${ }^{[20]}$. 此外, $\mathrm{SnCl}_{4}$ 在反应中可能发生水解生成相应 的 $\mathrm{Sn}$ 的水合物离子 [如 $\mathrm{Sn}\left(\mathrm{H}_{2} \mathrm{O}\right)_{n}(\mathrm{OH})_{y}{ }^{(4-y)+}$, 但这种水 解并不影响其糖类化合物的异构化的能力. 因此, Sn 离 子的总量在反应体系中是恒定的.

\section{4 水的添加量和液固比对反应的影响}

在纤维素醇解过程中, 少量水的添加有助于纤维素 的顺利解聚. 因此, 我们研究了水的加入量对于反应的 影响. 如图 4 所示, 在不添加水的情况下, 可以得到大 约 37.9\%的 ML. 其中 $\mathrm{SnCl}_{4}$ 催化剂中带有部分的结晶水 以及甲醇醚化产生的少量的水可能参与了纤维素的水 解过程. 随着向反应体系中加入水量的提高, ML 的产 率不断上升, 并在 $m_{\mathrm{H}_{2} \mathrm{O} / \text { cellulose }}=1.6$ (重量比) 时获得了最 高的产物产率. 而水量进一步增加时, 则可能影响葡萄 糖的转化途径, 生成更多腐殖质, 从而降低产物产 率 ${ }^{[24]}$.

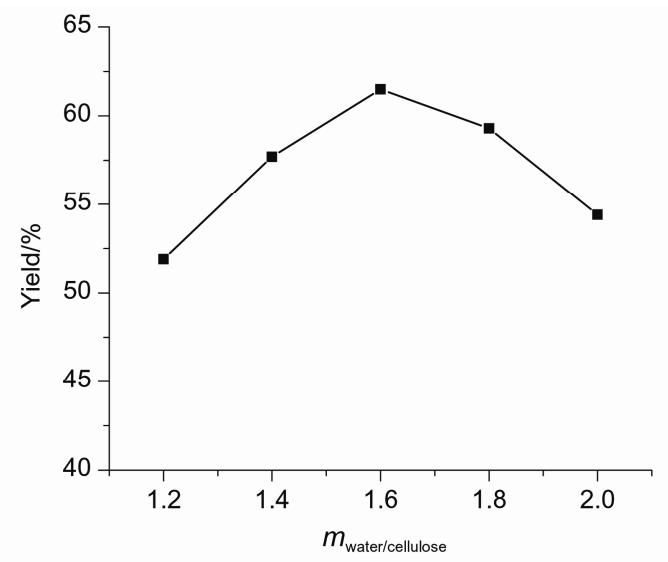

图 4 水的添加量对反应的影响

Figure 4 Influence of the amount of $\mathrm{H}_{2} \mathrm{O}$

此外, 反应溶剂与纤维素用量的比例(液固比)对反 应的影响也进行了研究. 在保持催化剂和纤维素的比例 恒定情况下, 随着液固比从 $16: 1$ 升至 $28: 1, \mathrm{ML}$ 产率 逐渐增加, 直至达到最大值 $61.5 \%$. 且反应液固比越高, 反应的残渣量越低. 结果表明高浓度的纤维素和催化剂 可能导致溶液酸强过大, 从而在微波条件下容易引发碳 化反应. 随着液固比的继续增加, 反应的产率急剧下降. 这可能是由于催化剂的浓度减少而导致的酸强减小, 影 响催化纤维素醇解的效率. 由此可见, 合适的液固比对 于反应的高效转化至关重要.

\section{5 温度对反应的影响}

从图 5 中可以看出, 当反应在较低温度进行时(如 $\left.150{ }^{\circ} \mathrm{C}\right), \mathrm{ML}$ 的产率仅有 $9.3 \%$. 随着温度的升高, ML 的 产率进一步升高, 当反应温度为 $180{ }^{\circ} \mathrm{C}$ 时, $\mathrm{ML}$ 的产率
达到最高. 继续提高反应温度, 则导致 ML 产率的下降. 对于固体残渣, 随着温度的上升, 其质量先慢慢减少 (纤维素转化程度提高). 当温度达到 $180{ }^{\circ} \mathrm{C}$ 时, 固体残 渣量最少. 继续提高温度至 $190{ }^{\circ} \mathrm{C}$ 时, 固体残渣开始增 加. 以上结果表明, 在纤维素醇解过程中, 需要综合考 虑反应效率和副反应发生程度这两个方面，以便获得最 高的产物产率 ${ }^{[25]}$.

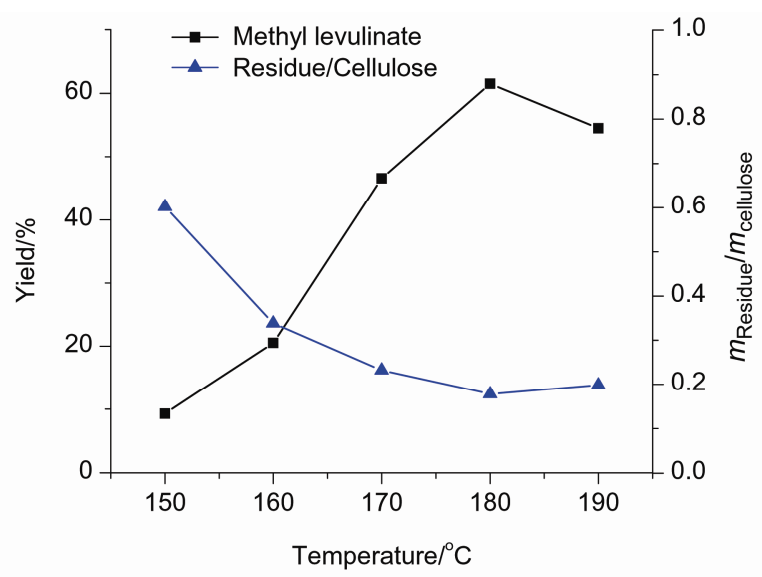

图 5 反应温度对反应的影响

Figure 5 Influence of the reaction temperature

\section{6 微波加热与常规加热对纤维素醇解效率的影响}

为了考察微波加热和常规加热对反应的影响, 我们 对两种条件下的反应分别进行了跟踪. 首先考察了微波 条件下不同反应时间所对应的产物分布情况(图 6a). 随 着反应进行, ML 产率慢慢提高, 固体残渣慢慢减少(反 应初始, 大量纤维素没来得及转化). 当时间为 $50 \mathrm{~min}$ 时，反应达到最高产率 $61.5 \%$. 进一步增加反应时间， 少量乙酰丙酸甲酯发生副反应而产率会慢慢下降，反应 体系中则生成越来越多的固体残渣 ${ }^{[18]}$. 同时, 对常规加 热下纤维素醇解反应也进行了跟踪(如图 6b). 保持其它 反应条件一致, 常规加热条件下反应 $50 \mathrm{~min}$ 后仅获得了 $29.5 \%$ 的 ML 产率. 延长时间至 $120 \mathrm{~min}$ 得到了 $57.5 \%$ 的 最高产率. 与微波加热方式对比可以看出, 微波加热提 高反应的速率，使得副反应也有所抑制，且两种加热方 式下 ML 的产率相差不大. 总体来看,, $\mathrm{SnCl}_{4} / \mathrm{H}_{2} \mathrm{SO}_{4}$ 二 元体系不仅能在微波条件下取得较高的乙酰丙酸甲酯 产率，在传统的高压反应釜加热方式下也有较好的效 果 ${ }^{[14]}$.

\section{7 其他生物质原料转化制备乙酰丙酸甲酯}

为了验证该催化体系的普适性, 我们选用了其他生 物质基糖类化合物进行了反应. 在没有进一步优化反应 条件的基础上，单糖、二糖和多糖得到 ML 的产率均在 $48 \%$ 以上. 其中，以葡萄糖为原料得到的 $\mathrm{ML}$ 产率为 $62.3 \%$, 纤维二糖为 $55.7 \%$, 蔗糖 $52.9 \%$. 多糖如菊粉、 


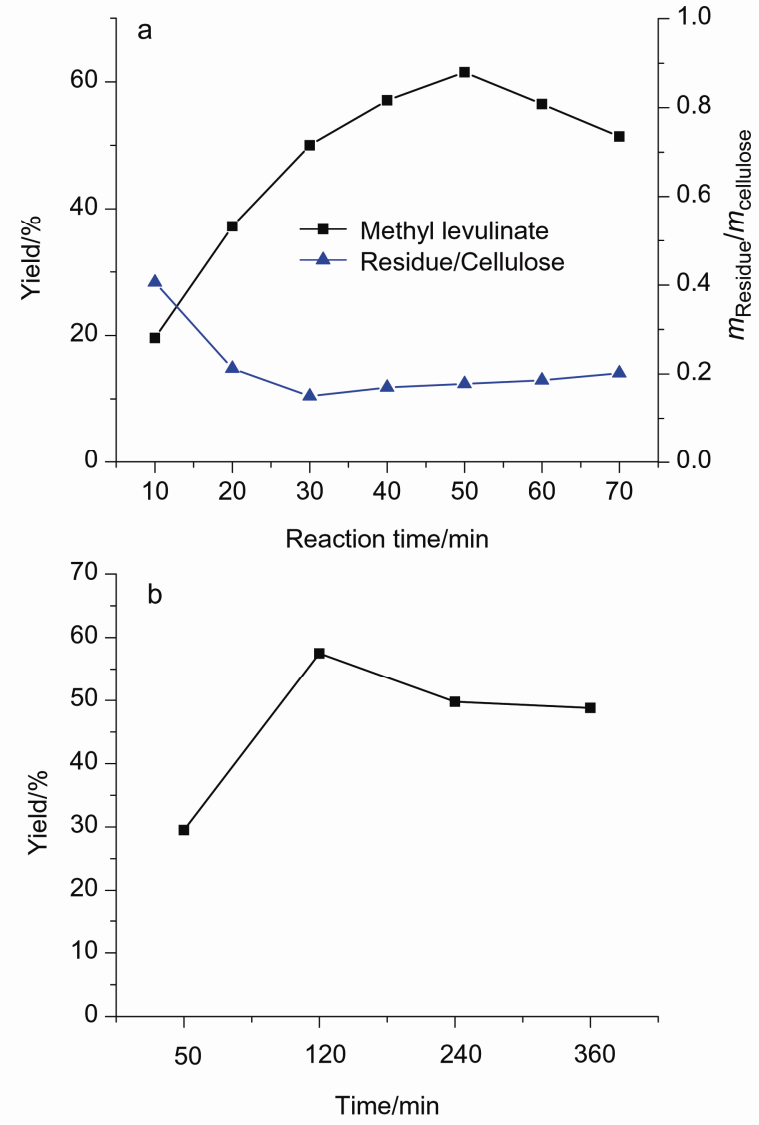

图 6 微波加热(a)和常规加热(b)对反应影响对比

Figure 6 Comparison of the heating methods microwave heating (a) conventional heating (b)

淀粉等作为起始原料时，也获得了 $48.1 \%$ 和 $56.6 \%$ 的 ML 产率. 结果这说明了该混合酸体系能很好地应用于多种 糖类化合物. 同时，我们对这些糖类反应条件进行了简 单优化，获得了更高产率的目标产物. 总的来说, $\mathrm{SnCl}_{4}$ 和 $\mathrm{H}_{2} \mathrm{SO}_{4}$ 组成的混合体系较好地适用于各类生物质基 碳水化合物的醇解.

表 2 不同碳水化合物制备乙酰丙酸甲酯的反应效果 ${ }^{a}$

Table 2 The conversion of different carbohydrates to methyl levulinate

\begin{tabular}{cccc}
\hline Entry & Substrate & ML yield ${ }^{b} \%$ & Methyl lactate yield ${ }^{b} \%$ \\
\hline 1 & Glucose & $60.3(52.3)$ & 9.1 \\
2 & Cellobiose & $55.7(48.6)$ & 11.8 \\
3 & Sucrose & $52.9(43.5)$ & 14.2 \\
4 & Inulin & $48.1(40.3)$ & 19.7 \\
5 & Starch & $56.6(50.8)$ & 9.9 \\
\hline
\end{tabular}

conditions: carbohydrate $3.1 \mathrm{mmol}, \mathrm{SnCl}_{4} 0.2 \mathrm{mmol}, \mathrm{H}_{2} \mathrm{SO}_{4} 0.7 \mathrm{mmol}, \mathrm{H}_{2} \mathrm{O}$ $0.8 \mathrm{~mL}, \mathrm{MeOH} 14 \mathrm{~mL}, 800 \mathrm{~W}, 180{ }^{\circ} \mathrm{C}, 50 \mathrm{~min} ;{ }^{b} \mathrm{GC}$ yield and isolated yield (in the parentheses).

\section{8 催化体系的循环使用}

为了研究催化剂的循环利用特性, 我们对反应的催 化剂进行回收和再利用. 反应后, 利用减压蒸馏除去甲 醇并得到含有催化剂和产物的粘稠物, 加水溶解后再用
二氯甲烷将有机物萃取分离, 收集水层后浓缩，即可分 离出催化剂粗品，直接用于下次反应. 催化剂循环 5 次 使用结果见表 3. 随着循环次数增加, ML 的产率稍有降 低，这可能是在催化剂回收的过程中，不可避免地产生 了少量的损失. 但从整体来看，回收后的催化剂仍能较 好地完成纤维素的催化转化反应.

表 3 催化剂循环使用

Table 3 Recycling of the catalyst

\begin{tabular}{cccccc}
\hline Run & 1 & 2 & 3 & 4 & 5 \\
\hline $\mathrm{ML} \mathrm{yield}^{b} \%$ & 61.5 & 59.3 & 58.1 & 57.0 & 54.9 \\
\hline
\end{tabular}

${ }^{a}$ Conditions: cellulose $500 \mathrm{mg}, 0.8 \mathrm{~mL} \mathrm{H} \mathrm{H}_{2} \mathrm{O}\left(m_{\mathrm{H}_{2} \mathrm{O} / \text { cellulose }}=1.6\right), 14 \mathrm{~mL}$ $\mathrm{MeOH}, 800 \mathrm{~W}, 180{ }^{\circ} \mathrm{C}, 50 \mathrm{~min} .{ }^{b} \mathrm{GC}$ yield.

\section{2 结论}

使用质子酸 $\mathrm{H}_{2} \mathrm{SO}_{4}$ 和路易斯酸性金属盐 $\mathrm{SnCl}_{4}$ 的二 元混合体系完成了纤维素到乙酰丙酸甲酯的高效转化. 研究结果表明: 在 $6.7 \mathrm{~mol} \% \mathrm{SnCl}_{4}, 23.3 \mathrm{~mol} \% \mathrm{H}_{2} \mathrm{SO}_{4}$, $180{ }^{\circ} \mathrm{C}, 50 \mathrm{~min}$ 和 $0.8 \mathrm{~mL}$ 水的条件下, 获得了 $61.5 \%$ 乙 酰丙酸甲酯的产率。同时，该体系还被应用到多种碳水 化合物醇解制备乙酰丙酸甲酯的反应中，获得了 $48 \%$ 以 上的产物产率. 该催化体系循环利用 5 次能仍能保持了 良好的催化活性. 该体系工艺简单，反应时间短，催化 剂易于回收, 为纤维素制备乙酰丙酸甲酯的生产提供了 一种新的方法.

\section{3 实验部分}

\section{1 试剂与仪器}

微晶纤维素(Sigma-Aldrich, 约 $20 \mu \mathrm{m}$ )、氯化盐类 (如 $\mathrm{SnCl}_{4} \cdot 5 \mathrm{H}_{2} \mathrm{O}$ 等)、液体酸(如硫酸、盐酸等)和其他糖 类化合物(如葡萄糖、果糖等)购自国药化学试剂有限公 司, 分析纯. 杂多酸杂多酸 $\left(\mathrm{H}_{3} \mathrm{O}_{40} \mathrm{PW}_{12} \cdot x \mathrm{H}_{2} \mathrm{O}\right.$ 和 $\mathrm{H}_{4}[\mathrm{Si}-$ $\left.\left.\left(\mathrm{W}_{3} \mathrm{O}_{10}\right)_{4}\right] \cdot x \mathrm{H}_{2} \mathrm{O}\right)$ 购自阿拉丁化学试剂有限公司. 其他所 用试剂均购自国药化学品集团, 分析纯. 气相色谱仪型 号是美国 Agilent 7890A, FID 检测器.

\section{2 实验方法}

纤维素原料制备乙酰丙酸酯的过程: 将 $500 \mathrm{mg}$ 的 微晶纤维素和 $14 \mathrm{~mL}$ 的醇加入到 $100 \mathrm{~mL}$ 的聚四氟乙烯 反应釜中，随后将 $0.2 \mathrm{mmol}$ 的 $\mathrm{SnCl}_{4} 、 0.7 \mathrm{mmol}$ 的 $\mathrm{H}_{2} \mathrm{SO}_{4}$ 和 $0.8 \mathrm{~mL}$ 水加入到反应釜中并进行密封. 在微波功率 为 $800 \mathrm{~W}$ 条件下加热 $2 \mathrm{~min}$ 以升温至目标温度并反应 50 $\min$ (定义反应体系被加热到目标温度的时刻为反应的 零时刻). 反应结束后, 加入 $0.3 \mathrm{mmol}$ 的萗作为内标并 通过气相色谱来计算产物的产率. 色谱条件如下: DB-5 (30 m×0.25 mm $\times 0.25 \mu \mathrm{m}$, Agilent)毛细管色谱柱; 初 温 $80{ }^{\circ} \mathrm{C}$ 保留 $2 \mathrm{~min}, 10{ }^{\circ} \mathrm{C} / \mathrm{min}$ 升温至 $200{ }^{\circ} \mathrm{C}$ 保留 3 
$\min$; 进样口温度 $250{ }^{\circ} \mathrm{C}$, 检测器温度 $250{ }^{\circ} \mathrm{C}$; 载气为 高纯氮气, 流速 $0.9 \mathrm{~mL} / \mathrm{min}$; 进样量为 $0.4 \mu \mathrm{L}$, 分流比 为 $50: 1$.

产物分离方法: 反应体系冷却后, 离心去除混合液 中的固体残渣. 得到粘稠液体后, 加入一定量的水溶解. 再往圆底烧瓶中加入二氯甲烷, 并转移至分液漏斗中萃 取, 最后获得二氯甲烷的萃取液. 将萃取液进行减压蒸 馏即可获得乙酰丙酸甲酯.

催化剂回收实验：将反应液离心除去固体残渣, 并 在 $50{ }^{\circ} \mathrm{C}$ 下除去甲醇溶剂. 得到深褐色粘稠液体后, 在 烧瓶中分次加入蒸馏水 $(10 \mathrm{~mL} \times 3)$ 震荡并转移到分液漏 斗中. 用 $20 \mathrm{~mL}$ 二氯甲烷萃取, 保留上层液体, 重复萃 取操作三次. 得到的上层水相液体转入到旋蒸瓶中, 减 压蒸馏下除去水分, 留下剩余物. 最后, 加入 $14 \mathrm{~mL}$ 甲 醇溶解并转移到聚四氟乙烯反应釜中进行下次反应.

辅助材料(Supporting Information) 目标产物乙酰丙 酸甲酯的 GC, GC-MS, ${ }^{1} \mathrm{H}$ NMR 和 ${ }^{13} \mathrm{C}$ NMR 谱图. 不同 反应条件对反应产率的影响. 这些材料可以免费从本刊 网站(http://sioc-journal.cn/)上下载.

\section{References}

[1] Chen, X.; Han, Z. Q.; Kong, F. H.; Hu, X. T. Prog. Chem. 2007, 19, 1091 (in Chinese). (陈曦, 韩志群, 孔繁华, 胡徐腾, 化学进展, 2007, 19, 1091.)

[2] Hu, L.; Sun, Y.; Lin, L. Prog. Chem. 2012, 24, 483 (in Chinese). (胡否, 孙勇, 林鹿, 化学进展, 2012, 24, 483.)

[3] Wu, S. C.; Wang, C. L.; Gao, Y. J.; Zhang, S. C.; Ma, D.; Zhao, Z. B. Chin. J. Catal. 2010, 31, 1157 (in Chinese). (吴树昌, 王春雷, 高勇军, 张少春, 马丁, 赵宋保, 催化学报, 2010, 31, 1157.)

[4] Peng, L. C.; Lin, L.; Li, H. Prog. Chem. 2012, 24, 801 (in Chinese). (彭林才，林鹿，李辉，化学进展, 2012, 24, 801.)

[5] Runge, T.; Zhang, C. Ind. Eng. Chem. Res. 2012, 51, 3265.
[6] Yang, Z.; Fu, Y.; Guo, Q. X. Chin. J. Org. Chem. 2015, 35, 273 (in Chinese). (杨珍, 傅尧, 郭庆祥, 有机化学, 2015, 35, 273.)

[7] Zhang, T.; Chang, C. Chem. Ind. Eng. Prog. 2012, 31, 1224 (in Chinese). (张婷, 常春, 化工进展, 2012, 31, 1224.)

[8] Joshi, H.; Moser, B. R.; Toler, J.; Smith, W. F.; Walker, T. Biomass Bioenergy 2011, 35, 3262.

[9] Chia, M.; Dumesic, J. A. Chem. Commun. 2011, 47, 12233.

[10] Huang, Y.; Dai, J.; Deng, X.; Qu, Y.; Guo, Q.; Fu, Y. ChemsusChem 2011, 4, 1578.

[11] Alexandre, D.; Nadine, E.; Franck, R. ACS Sustainable Chem. Eng. 2014, 2, 1338.

[12] Climent, M. J.; Corma, A.; Iborra, S. Green Chem. 2014, 16, 516.

[13] Melero, J. A.; Morales, G.; Iglesias, J.; Paniagua, M.; Hernandez, B.; Penedo, S. Appl. Catal., A 2013, 466, 116.

[14] Huang, Y. B.; Yang, T.; Zhou, M. C.; Pan, H.; Fu, Y. Green Chem. 2016, 18, 1516.

[15] Liu, D.; Lin, L.; Zeng, S. S.; Peng, L. C. Chem. Ind. Forest Prod. 2013, 33, 66 (in Chinese). (刘娣，林鹿，曾姗姗，彭林才，林产化学与工业, 2013, 33, 66.)

[16] Yu, K. M. K.; Tsang, S. C. Catal. Lett. 2011, 141, 259.

[17] Tominaga, K.; Mori, A.; Fukushima, Y.; Shimada, S.; Sato, K. Green Chem. 2011, 13, 810.

[18] Ma, H.; Long, J. X.; Wang, F. R.; Wang; L. F.; Li, X. H. Acta Phys. Chim. Sin. 2015, 31, 973 (in Chinese). (马浩, 龙金星, 王芙蓉, 王乐夫, 李雪辉, 物理化学学报, 2015, 31, 973.)

[19] Sun, P. Q.; Zhao, S. Q.; Chang, Q.; Chen, J. W. J. Zhengzhou Univ. 2014, 35, 22 (in Chinese). (孙培勤, 赵世强, 常春, 陈俊武, 郑州大学学报, 2014, 35, 22.)

[20] Ding, D. Q.; Xi, J. X.; Wang, J. J.; Liu, X. H.; Lu, G. Z.; Wang, Y. Q. Green Chem. 2015, 17, 4037.

[21] Hishikawa, Y.; Yamaguchi, M.; Kubo, S.; Yamada, T. J. Wood Sci. 2013, 59, 179.

[22] Li, J.; Huang, Y. B.; Guo, Q. X.; Fu, Y. Acta Chim. Sinica 2014, 72, 1223 (in Chinese). (李江，黄耀兵，郭庆祥，傅尧，化学学报, 2014, 72, 1223.)

[23] Wu, X.; Fu, J.; Lu, X. Carbohydr. Res. 2012, 358, 37.

[24] Chang, C.; Jiang, X. X.; Zhang, T. Adv. Mater. Res. 2012, 512, 388.

[25] Peng, L.; Lin, L.; Li, H.; Yang, Q. Appl. Energ. 2011, 88, 4590. 